\title{
VARIABILIDAD Y CORRELACIÓN EN LA CONCENTRACIÓN DE LÍPIDOS SANGUíNEOS EN DOS LÍNEAS GENÉTICAS DE GALLINAS PONEDORAS
}

\begin{abstract}
VARIABILITY AND CORRELATION OF BLOOD LIPIDS CONCENTRATION IN TWO GENETIC LINES OF LAYING HENS
\end{abstract}

\author{
Jancy Darly Flórez ${ }^{1}$, José Henry Osorio², Jorge Enrique Pérez ${ }^{3}$
}

\begin{abstract}
${ }^{1}$ DVM. M.Sc. Ciencias Veterinarias, Departamento de Salud Animal, Universidad de Caldas, Manizales, Colombia jancydarly@ yahoo.com.ar; ${ }^{2}$ DVM, M.Sc. Bioquímica. Ph.D. Bioquímica Clínica y Patología Molecular. Laboratorio de Bioquímica Clínica y Patología Molecular, Departamento de Ciencias Básicas de la Salud, Universidad de Caldas, Manizales, Colombia: jose. osorio_o@ucaldas.edu.co; ${ }^{3}$ BSc.M.Sc. Microbiología, laboratorio de Microbiología, Universidad de Caldas, Manizales, Colombia: labmicro@ucaldas.edu.co
\end{abstract}

Rev. U.D.CA Act. \& Div. Cient. 16(1): 151 - 158, 2013

\section{RESUMEN}

En este estudio, se evaluaron y se correlacionaron los niveles de colesterol total, triglicéridos y colesterol de la lipoproteína de alta densidad en dos líneas de gallinas ponedoras y además, se tuvo en cuenta la producción de huevo, como trabajo inicial en la investigación, variabilidad del perfil lipídico en las gallinas ponedoras, en las diferentes etapas de producción. Se obtuvo suero de 90 gallinas ponedoras en ayunas, de 26 semanas de edad, 45 Hy-Line-W-36 y 45 Lohmann Brown-Classic. Se determinó la concentración de colesterol, triglicéridos y colesterol lipoproteína de alta densidad, mediante métodos enzimáticos colorimétricos. Las medias \pm error estándar en $\mathrm{mg} / \mathrm{dL}$, para los niveles de colesterol total, triglicéridos y colesterol de la lipoproteínas de alta densidad, para la línea Hy-Line W-36 fueron de: 141,8 $\pm 5,49$; $773,2 \pm 48,06$; 13,4 \pm 052 ; respectivamente y para la línea Lohmann Brown-Classic fueron de: $111,1 \pm 3,58 ; 431,7 \pm 28,74$; $16,6 \pm 0,4$, respectivamente. Todas las variables presentaron diferencias significativas $(\mathrm{P}<0,05)$. Se encontró relación positiva $(P<0,05)$ entre el colesterol total con los triglicéridos en las dos líneas y entre el colesterol total con el colesterol de la lipoproteína de alta densidad en la línea Lohmann BrownClassic. La línea Hy-Line W-36 mostró mayores niveles de colesterol total, triglicéridos y la Lohmann Brown-Classic mayores niveles de colesterol de la lipoproteína de alta densidad y mayor producción de huevo, por lo tanto, se hace necesario realizar trabajos para conocer cómo repercutirían los niveles elevados de triglicéridos y colesterol en el huevo en aves, con una producción menor a la esperada y sus implicaciones en la salud humana.
Palabras clave: Colesterol, HDL, gallinas de postura, metabolismo, triglicéridos.

\section{SUMMARY}

This study evaluated and correlated the levels of total cholesterol, triglycerides and high density lipoprotein cholesterol in two laying hens lines; taking also into account the egg production, as initial work in the investigation, variability lipid profile in laying hens at different stages of production. After fasting, serum of 90 laying hens 45 Hy-Line W-36 y 45 Lohmann Brown-Classic of 26 weeks of age was taken. The levels of the total cholesterol, triglycerides and high density lipoprotein cholesterol were determined using the enzymaticcholorimetric method. Mean \pm standard error for total cholesterol, triglycerides and high density lipoprotein cholesterol for Hy-Line W-36 line in mg/dL were: 14.,8 $85.49 ; 773.2 \pm 48.06$; 13.4 \pm 052 ; respectively, and Lohmann Brown-Classic line in $\mathrm{mg} / \mathrm{dL}$ were: $111.1 \pm 3.58$; $431.7 \pm 28.74 ; 16.6 \pm 0.4$; respectively. There were differences $(P>0.05)$ among lines for levels of total cholesterol, triglycerides and high density lipoprotein cholesterol. Total cholesterol was positively correlated with triglycerides in two lines, and total cholesterol with the high density lipoprotein cholesterol in the Lohmann Brown-Classic line, with significance $(P<0.05)$. It was observed that Hy-Line W-36 line has higher levels of cholesterol and triglycerides, and the Lohmann Brown-Classic line has more levels of high density lipoprotein cholesterol and higher egg production, which makes it necessary to perform further research to determine how high levels of cholesterol and triglycerides within eggs have repercussions, with a decrease 
in the expected production, and its implication in human health.

Key words: Cholesterol, HDL, laying hens, metabolism, triglycerides.

\section{INTRODUCCIÓN}

Los lípidos son biomoléculas orgánicas importantes en la alimentación humana y animal. Se caracterizan por ser hidrofóbicos, por lo que necesitan estar unidos a proteínas, específicamente, apolipoproteínas, para ser transportados por el torrente sanguíneo (Murray et al. 2004). Esta biomolécula es la principal fuente de energía metabólica, se oxida para producir ATP y es depositada en el tejido adiposo como triglicérido (Montgomery \& Conway, 1998) y en la yema de huevo de las aves, como triglicérido, colesterol y fosfolípido (Walzem et al. 1999).

En este sentido, las variaciones en la concentración de estos lípidos y de las apolipoproteínas constituyen las lipoproteínas, las cuales, se clasifican en: portomicrones (quilomicrones en mamíferos) (Noyan et al. 1964); lipoproteínas de muy baja densidad (VLDL, del inglés: Very Low density lipoprotein); lipoproteínas de densidad intermedia (IDL, del inglés: Intermediate density lipoprotein), lipoproteínas de baja densidad (LDL, del inglés: Low density lipoprotein) y lipoproteínas de alta densidad (HDL, del inglés: High density lipoprotein) (Hermier, 1997; Hermier et al. 1984; Newsholme \& Leech, 1987). Además, las gallinas tienen la vitelogenina (VTG), una lipofosfoproteína de alta densidad que con otras lipoproteínas forman parte de la yema del huevo (Hermier et al. 1989).

Las gallinas ponedoras, por acción de los estrógenos, presentan hipertrigliceridemia, sobretodo en la máxima producción de huevos (Kudzma et al. 1975), debido a cambios metabólicos en las VLDL, que inducen a la síntesis de la apolipoproteína VLDL-II (apoVLDL-II), la cual, impide la hidrolisis de las VLDL y la liberación de los triglicéridos por la acción de la lipoproteína lipasa (LPL); además, inducen a la formación de las VLDLy ("y" del inglés "yolk"), ricas en triglicéridos, pero de menor tamaño, importante para facilitar la entrada al oocito (Walzem et al. 1999).

Las VLDL que no tienen la apoVLDL-II son hidrolizadas por la LPL y convertidas en LDL (ricas en colesterol esterificado y libre), a su vez, son captadas por las células somáticas, para mantener la homeostasis del colesterol, de forma similar a los mamíferos (Nimpf \& Schneider, 1991).

Los estrógenos también afectan los niveles de lipoproteína de alta densidad (HDL), pero disminuyen su concentración en plasma; sin embargo, esta lipoproteína que también entra al oocito en crecimiento, incorpora colesterol libre a la yema
(Vieira et al. 1995). Cabe mencionar que las gallinas ponedoras se caracterizan por la aparente ausencia del patrón lipoproteína (LDL o HDL), común en mamíferos y en aves inmaduras (Hermier et al. 1989).

Los lípidos en las aves han sido estudiados y cuantificados en su mayoría en ambientes controlados (Nimpf \& Schneider, 1991) y con diversos métodos analíticos, como ultracentrifugación de las lipoproteínas o Kits enzimáticos colorimétricos (An et al. 1997), los cuales, se basan en la medición del colesterol que transportan las lipoproteínas, medidas por ultracentrifugación.

Los investigaciones muestran que los niveles lipídicos en las gallinas varían entre las líneas genéticas, dependiendo de la cantidad del aditivo utilizado en la dieta (Yin et al. 2008); además, algunos aditivos en las dietas pueden llegar a disminuir los lípidos en la sangre de las gallinas ponedoras, hasta el punto de disminuir la producción de huevo (König et al. 2007).

También, se ha estudiado la correlación entre los lípidos sanguíneos y los lípidos incorporados a la yema de huevo (Chowdhury et al. 2002; Chowdhury et al. 2005; König et al. 2007; Salma et al. 2007; Vieira et al. 1995; Yin et al. 2008). Esta estructura del huevo posee, aproximadamente, $190 \mathrm{mg}$ de colesterol por huevo (Liu et al. 2010; Anderson, 2011) y de triglicérido, cerca de 3100 mg/huevo (Salma et al. 2007). Estos lípidos, que en exceso son perjudiciales, han provocado la disminución del consumo de huevo, ya que solamente es recomendable el consumo de $300 \mathrm{mg}$ de colesterol diarios en hombres y en mujeres adultos sanos (Weggemans et al. 2001); no obstante, en cuanto a los triglicéridos, los niveles sanguíneos en las personas se ven alterados no únicamente por el consumo directo de lípidos y de carbohidratos, sino por el ejercicio físico, entre otros.

El objetivo del presente trabajo fue comparar la concentración sérica de colesterol total, triglicéridos y colesterol lipoproteína de alta densidad y su interacción en dos líneas genética de gallina ponedora, mediante métodos enzimáticos colorimétricos.

\section{MATERIALES Y MÉTODOS}

Animales y dieta: La investigación, se desarrolló en la "Granja Montelindo", propiedad de la Universidad de Caldas, Colombia, a una temperatura entre 23 y $30^{\circ} \mathrm{C}$. Un total de 90 gallinas ponedoras (45 Hy-Line W-36 y 45 Lohmann BrownClassic) fueron criadas, según diseño completamente al azar, durante las semanas 25 y 26 de edad, en pleno pico de puesta. Las gallinas se mantuvieron a régimen de 13 horas de luz.

El galpón estaba subdividido en 40 jaulas de piso (20 jaulas 
por línea), el tamaño era de 2,5m de ancho x 2,5m de largo x $3 \mathrm{~m}$ de alto; cada jaula poseía un nidal, dos comederos y un bebedero. Diariamente, se recogían los huevos y se cuantificaban por jaula. Para ser posible el presente ensayo, se escogieron dos jaulas, una para cada 45 gallinas, según la línea.

La dieta fue formulada a base de maíz y torta de soya. Para la dieta de las dos líneas utilizadas, nos basamos en un trabajo donde analizan cinco dietas para tres fases diferentes en dos líneas de gallina ponedora; en dicho trabajo, se basan en los manuales para la recomendación de los nutrientes, pero sin hacer distinción para cada línea (Novak \& Scheideler, 2001) (Tabla 1). Se les administró durante las semanas 25 y 26: 90g/ave/día, para las gallinas Hy-Line w-36, las cuales, llegaron a un peso de $1.444 \mathrm{~g} /$ ave y $110 \mathrm{~g} /$ ave/día, para las gallinas Lohmann Brown-Classic, que llegaron a un peso de $1.851 \mathrm{~g} /$ ave. El agua fue administrada ad libitum.

Tabla 1. Composición nutricional de la dieta para gallinas ponedoras $(\mathrm{kg} / 100 \mathrm{~kg})$.

\begin{tabular}{|l|r|}
\hline Ingredientes & kg \\
\hline Maíz & 59,120 \\
\hline Salvado de trigo & 2,646 \\
\hline Torta de soya (48 \%) & 25,716 \\
\hline DL- metionina & 0,220 \\
\hline L- treonina & 0,055 \\
\hline Carbonato de calcio & 8,628 \\
\hline Dicalfos (fosfato bical) & 1,819 \\
\hline Aceite de soya & 1,250 \\
\hline Colina & 0,200 \\
\hline Premezcla de vitaminas y minerales & 0,100 \\
\hline Sal & 0,250 \\
\hline
\end{tabular}

Composición calculada:

-Proteína cruda, \%

-Energía metabolizable, kcal/kg

2748,97

-Extracto etéreo, \%

-Metionina, \%

0,45

-Lisina, \%

0,90

-Triptofano, \%

0,20

-Treonina, \%
Recolección de la muestra: Antes de la toma de muestras, las aves tuvieron $16 \mathrm{~h}$ de ayuno, se tomó como referencia un trabajo, donde analizaron lípidos sanguíneos en gallinas ponedoras con un ayuno de 15h (Elkin et al. 1993); se extrajeron $5 \mathrm{~mL}$ de sangre directamente de la vena yugular, en tubos de ensayo. La sangre, se centrifugó (Hettich Zentrifugen, EBA 20. Tuttlingen, Germany) a 3000 revoluciones por minuto (rpm), durante 15 minutos, a temperatura ambiente; seguido el suero obtenido, se congeló a $-30^{\circ} \mathrm{C}$, hasta su posterior análisis. Al momento de procesar las muestras, los sueros fueron descongelados a temperatura ambiente $y$, luego, fueron depositados en baño maría, a una temperatura de $37^{\circ} \mathrm{C}$, por 10 minutos.

Métodos de análisis: Todos los reactivos pertenecían a los laboratorios BioSystems S.A., Barcelona, España, la concentración de colesterol libre, triglicéridos y C-HDL, fueron determinados por métodos enzimáticos-colorimétricos, de acuerdo a las recomendaciones del fabricante. Los análisis fueron realizados en un equipo RAYTO RT-1904C (Rayto Life and Analytical Sciences Co., Ltd. Shenzhen, China), analizador semiautomático de química.

Determinación del colesterol total (colesterol libre + esteres de colesterol): La determinación del CT en suero, se realizó mezclando $10 \mu \mathrm{L}$ de la muestra y $1 \mathrm{~mL}$ de reactivo $(\mathrm{Pi}-$ pes $35 \mathrm{mmol} / \mathrm{L}$, colato sódico $0,5 \mathrm{mmol} / \mathrm{L}$, fenol $28 \mathrm{mmol} / \mathrm{L}$, colesterol esterasa $>0,2 U / \mathrm{mL}$, colesterol oxidasa $>0,1 U / \mathrm{mL}$, peroxidasa $>0,8 \mathrm{U} / \mathrm{mL}, 4$-amino antipirina (4AA) $0,5 \mathrm{mmol} / \mathrm{L}$, $\mathrm{pH} 7,0$ ). Seguido, se agitó manualmente por 5 segundos la mezcla y se dejó incubar en los tubos de ensayo, durante 10 minutos, a temperatura ambiente. Los ésteres de colesterol fueron hidrolizaros por la colesterol esterasa, dando lugar al colesterol libre, que por acción de la colesterol oxidasa formó colestenona + peróxido de hidrógeno, este último, en presencia de la 4-AA y fenol dieron lugar a la quinonaimina, por acción de la peroxidasa. La quinonaimina es proporcional al colesterol total de la muestra y se cuantificó espectrofotométricamente.

Determinación de triglicéridos: Para determinar los TAG en suero fueron utilizados $10 \mu \mathrm{L}$ de la muestra y $1 \mathrm{~mL}$ de reactivo (Pipes $45 \mathrm{mmol} / \mathrm{L}$, 4-clorofenol $6 \mathrm{mmol} / \mathrm{L}$, cloruro magnésico $5 \mathrm{mmol} / \mathrm{L}$, lipasa $>100 \mathrm{U} / \mathrm{mL}$, glicerol-quinasa $>1,5 \mathrm{U} / \mathrm{mL}$, glicerol-3P-oxidasa $>4 \mathrm{U} / \mathrm{mL}$, peroxidasa $>0,8 \mathrm{U} / \mathrm{mL}$, 4-AA 0,75mmol/L, ATP 0,9mmol/L, pH 7,0). Se agitó manualmente la mezcla por 5 segundos y se dejó incubar los tubos durante 15 minutos, a temperatura ambiente. En el anterior proceso, los triglicéridos fueron hidrolizados por la lipasa a glicerol y ácidos grasos; el glicerol, en presencia de ATP fue fosforilado por la glicerol-quinasa y dio lugar al glicerol 3P +

0,75 ADP; el glicerol 3P en presencia de oxígeno formó peróxido de hidrógeno, por acción de la glicerol-3P-oxidasa; finalmente, se cuantificó espectrofotométricamente la quinonaimina 
producto de la acción de la peroxidasa sobre el peróxido de hidrógeno en presencia de 4-AA y clorofenol; la quinonaimina es proporcional a la concentración de los TAG.

Determinación del colesterol HDL mediante el método de precipitado (Fosfotungstato): Para realizar este método fue mezclado $1 \mathrm{~mL}$ de reactivo (Fosfotungstato $0,4 \mathrm{mmol} / \mathrm{L}$ y cloruro de magnesio $20 \mathrm{mmol} / \mathrm{L}$ ), con $0,2 \mathrm{~mL}$ de la muestra de suero; seguido, se agitó manualmente por 5 segundos y se dejó durante 10 minutos, a temperatura ambiente y se centrifugó por 10 minutos a 4000rpm. En el precipitado quedaron las VLDL, IDL y LDL, y en el sobrenadante las HDL.

Finalmente, se tomó $100 \mu \mathrm{L}$ del sobrenadante que fue depositado en otro tubo de ensayo, se mezcló con $1 \mathrm{~mL}$ del reactivo para colesterol total (descrito anteriormente) y se incubó por 10 minutos, en baño maría, a $37^{\circ} \mathrm{C}$. El colesterol HDL fue hidrolizado por la colesterol esterasa y la colesterol oxidasa, esto dio lugar a peróxido de hidrógeno, que fue consumido por una peroxidasa en presencia de la 4-AA y fenol, quedando como producto final la quinonaimina, siendo este producto proporcional al colesterol HDL de la muestra que fue cuantificado por espectrofotométria.
Los datos fueron analizados usando ANOVA simple. Las diferencias estadísticamente significativas, se determinaron con el $\mathrm{P}$ valor $<0,05$ del test $\mathrm{F}$. Se evaluó el coeficiente de correlación de Pearson entre colesterol total, triglicéridos y colesterol HDL con una significancia $P<0,05$. Para el análisis estadístico se utilizó Statgraphics Plus 5.1.

\section{RESULTADOS Y DISCUSIÓN}

El cambio en la composición nutricional de la dieta en la semana 25 redujo la producción de huevo, de 98 a 96\%, en la línea Lohmann Brown-Classic; sin embargo, la línea HyLina W-36 aumentó su producción, de 94 a 96\%. En la semana 26 , después de la adaptación al alimento, las gallinas Lohmann Brown recuperaron su porcentaje de producción, de $98 \%$ y las Hy-Line lo mantuvieron, en un $96 \%$ (Tabla 2); no obstante, solamente hubo diferencias significativas entre líneas en la semana 26 (Tabla 3).

En la tabla 4, se observa la concentración lipídica sérica de dos líneas genéticas de gallinas ponedoras. La línea de gallinas ponedoras Hy-Line W-36 presentó las mayores concentraciones de colesterol total y triglicéridos; sin embargo, la concentración de colesterol HDL incrementó en la

Tabla 2. Porcentaje de producción de huevo de Hy-Line W-36 y Lohmann Brown-Classic.

\begin{tabular}{|l|c|c|c|c|}
\hline \multirow{2}{*}{ Semana de Producción } & \multicolumn{2}{|c|}{ Hy-Line W-36* } & \multicolumn{2}{c|}{ Lohmann B-C* } \\
\cline { 2 - 5 } & No. Huevos/semana & $\mathbf{\%}$ & No. Huevos /semana & $\%$ \\
\hline Semana 24 & 296 & 94 & 307 & 98 \\
\hline Semana 25** & 302 & 96 & 301 & 96 \\
\hline Semana 26** & 300 & 96 & 309 & 98 \\
\hline
\end{tabular}

*45 aves de cada línea

**Alimentados con la dieta a base de maíz y torta de soya (Tabla 1)

Nota: No se tiene en cuenta la producción total de los dos lotes dentro del galpón

Tabla 3. Efecto de la línea en la producción de huevo.

\begin{tabular}{|c|c|c|c|}
\hline \multirow{2}{*}{ Semana de Producción } & Hy-Line W-36* & \multicolumn{2}{|c|}{ Lohmann B-C* } \\
\hline & Media \pm ES & Media \pm ES & P-valor \\
\hline Semana 24 & 42,28 & 43,85 & 0,21 \\
\hline Semana $25^{* *}$ & 43,14 & 43,00 & 0,86 \\
\hline Semana $26 * *$ & 42,57 & 44,14 & 0,02 \\
\hline
\end{tabular}

*45 aves de cada línea

**Alimentados con la dieta a base de maíz y torta de soya (Tabla 1)

Nota: No se tiene en cuenta la producción total de los dos lotes dentro del galpón 
Tabla 4. Efecto de la línea en el colesterol total, triglicéridos, C- HDL.

\begin{tabular}{|l|c|c|c|c|c|}
\hline \multirow{2}{*}{ PARÁMETRO } & \multicolumn{2}{|c|}{ Hy-Line W-36 } & \multicolumn{2}{c|}{ Lohmann Brown-Classic } & \multirow{2}{*}{ P-valor } \\
\cline { 2 - 5 } & Media \pm ES (mg/dL) & CV (\%) & Media \pm ES $(\mathrm{mg} / \mathrm{dL})$ & $\mathrm{CV}(\%)$ & \\
\hline Colesterol total & $141,8 \pm 5,49$ & 25,995 & $111,1 \pm 3,58$ & 21,627 & $<0,001$ \\
\hline Triglicéridos & $773,2 \pm 48,06$ & 41,698 & $431,7 \pm 28,74$ & 44.660 & $<0,001$ \\
\hline C-HDL & $13,4 \pm 0,52$ & 25,936 & $16,6 \pm 0,40$ & 16,014 & $<0,001$ \\
\hline
\end{tabular}

${ }^{\mathrm{a}-\mathrm{b}}$ Letras diferentes indican diferencias significativas con $\mathrm{P}<0,05$

ES: Error estándar, C-HDL (Colesterol de lipoproteína de alta densidad)

$\mathrm{CV}$ : Coeficiente de variación

línea Lohmann Brown-Classic, con diferencias significativas $(P<0,05)$, entre tratamientos.

Los niveles séricos de colesterol total son similares (135 - 145mg/dL) a los trabajos reportados por An et al. (1997) y Salma et al. (2007); no obstante, otras investigaciones muestran variaciones en los niveles de colesterol en sangre con menores (64,3 - 91,8mg/dL) (Yin et al. 2008) y mayores (150-240mg/dL) (Chowdhury et al. 2002) concentraciones. Hay que señalar que estas variaciones pueden corresponder a la línea genética, al tipo de dieta o al kit enzimático utilizado para el análisis de las muestras de dichos trabajos.

En este estudio, las diferencias encontradas en la concentración del colesterol total entre líneas, coinciden a lo reportado por Chowdhury et al. (2002), cuando evaluó estirpes alimentadas con ajo, con el fin disminuir el colesterol de la yema; sin embargo, Yin et al. (2008) trabajando con dos tipos de gallinas ponedoras, indicaron solamente diferencias significativas, cuando interactúan estirpes y dietas con diferentes niveles de ácido linoleico conjugado.

Es posible, que la mayor o menor concentración de colesterol en la sangre de las gallinas ponedoras, no sea un indicativo específico en el porcentaje de colesterol en la yema de huevo, pues se presentan resultados contradictorios en diversas publicaciones, ya sea indicando una correlación del colesterol sanguíneo con el colesterol del huevo (Chowdhury et al. 2002) o negando la relación directa de estos dos parámetros (Chowdhury et al. 2005). Las características genéticas de las diferentes líneas de gallinas ponedoras y el empleo de aditivos o nutrientes hipocolesterolémicos en las dietas o a la interacción entre líneas y dieta pudieran influir en estos resultados.

Otro factor que influye en los niveles de colesterol sérico es la edad de la gallina, ya que un estudio realizado por Peebles et al. (2004), se encontró que las semanas de producción influyen en la redistribución del colesterol sérico de las diferentes lipoproteínas de las gallinas ponedoras; además, puede afectar el porcentaje de colesterol de la yema de huevo (Anderson, 2011).

Las diferencias observadas en los niveles de colesterol HDL entre las líneas Lohmann Brown Classic y Hy Line-W-36 del presente trabajo, coinciden con otra investigación, donde los autores presentan diferencias significativas entre dos líneas genéticas de gallinas ponedoras (Brown Dwarf y White Leghorn) (Yin et al. 2008); igualmente, indican que el colesterol en el huevo no depende, de manera directa, de los niveles de colesterol HDL en el suero; por consiguiente, los mayores niveles de colesterol HDL del suero en las gallinas Lohman Brown, no deberían afectar directamente la cantidad de colesterol en la yema de huevo de dicha línea. No obstante, esto contradice otro trabajo, donde los autores señalan que los niveles sanguíneos de HDL, influyen directamente con los niveles de HDL en la yema de huevo y, por lo tanto, con el colesterol que transportan (Vieira et al. 1995). Los datos contradictorios, se podrían deber, según Peebles et al. (2004), a las semanas de producción de las gallinas ponedoras.

El presente estudio muestra una correlación positiva y significativa entre los niveles de colesterol total y colesterol HDL $(\mathrm{P}<0,05)$ en la línea Lohmann Brown-Classic; sin embargo, se observó una relación negativa no significativa en la línea Hy line W-36 (Cuadro 1). Esto se pudo haber debido a que las HDL son las encargadas de transportar el colesterol al hígado para su eliminación biliar (Lagarrigue et al. 2000) o a la yema de huevo (Vieira et al. 1995). Los niveles menores en suero de colesterol HDL y la relación negativa encontrada entre colesterol total y el colesterol HDL en la línea Hy-Line W-36 podrían indicar que esta línea, en la semana 26 de producción, tiene una menor cantidad de colesterol total disponible para la yema de huevo. 
Cuadro 1. Coeficiente de correlación de las variables colesterol total (CT), triglicéridos (TAG), lipoproteínas de alta densidad (HDL).

\begin{tabular}{|c|c|c|c|c|}
\hline \multirow{4}{*}{ 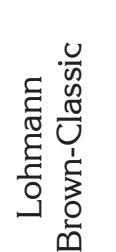 } & \multicolumn{4}{|c|}{ Hy-Line W-36 } \\
\hline & Parámetro & $\mathrm{CT}$ & TAG & C-HDL \\
\hline & CT & & $0,82 * *$ & $-0,05$ \\
\hline & TAG & $0,68 * *$ & & $-0,17$ \\
\hline & HDL & $0,49 *$ & 0,27 & \\
\hline
\end{tabular}

*Relación significativa $(P<0,05)$ relativamente débil

** Relación significativa $(\mathrm{P}<0,05)$ moderadamente fuerte

En las gallinas ponedoras, la acción de los estrógenos aumentan los niveles de triglicéridos y disminuyen los niveles de HDL (Hermier et al. 1989); no obstante, al analizar la relación de los triglicéridos con el colesterol HDL en esta investigación, solamente se observó una correlación negativa en la línea Hy-line W-36 y una correlación positiva con la línea Lohmann Brown-Classic, pero sin significancia para las dos líneas (Cuadro 1).

En el presente estudio, los niveles de triglicéridos indicaron diferencias significativas entre líneas (Tabla 4), lo que contrasta con otros trabajos, donde no se observan diferencias entre las líneas estudiadas (Yin et al. 2008); asimismo, se observó, que los niveles de triglicéridos mantuvieron un rango amplio, especialmente, en las gallinas Hy-Line W-36; esto pudiera explicar que en las gallinas ponedoras las lipoproteínas sérica más abundantes son las VLDLs, debido a que los triglicéridos son los principales componentes (Hermier, 1997; Hermier et al. 1989) y su concentración varía en dependencia a la concentración de las VLDLs en el plasma.

Por otra parte, diversos estudios muestran variaciones en la concentración de triglicéridos sanguíneos en gallinas ponedoras: An et al. (1997); Peebles et al. (2004); König et al. (2007); Salma et al. (2007) y Yin et al. (2008) han reportado estas variaciones lipídicas e indican que se pudiera deber al mejoramiento genético, condiciones medioambientales, semana de producción o dieta, entre otros.

En este sentido, la mayor concentración de triglicéridos séricos en las gallinas de la línea Hy-Line W-36 (Tabla 4) pudiera ser por la línea genética, ya que fue creada para incrementar y para mantener una alta producción de huevo (345-361 huevos, hasta la semana 80 de edad) (Hy-Line International 2009); sin embargo, una excelente producción, depende de una alta concentración de VLDL en plasma, pues cuando disminuye la concentración de VLDL en el torrente sanguíneo, se deprime la producción de huevo, aunque no influye en los triglicéridos de la yema (König et al. 2007), acción que lograron los autores, usando clofibrato (sustancia hipo- lipemiante).

No obstante, en el presente trabajo, es posible que la disminución de la producción de huevo en las gallinas Hy Line W-36 comparada con las gallinas Lohmann Brown-Classic, se deba a la excesiva manipulación o cambios de temperatura ambiental (Tabla 2); además, el lote de la línea Hy-Line W-36, de donde se adquirieron las aves, tenían solamente el 92\% y el lote de la línea Lohmann Brown-Classic un 97\% de producción en la semana 26 (datos no reportados). Pese a que los resultados del presente artículo, contradicen lo reportado por Mumma et al. (2006), donde indicaron en el estrés incrementa los niveles de colesterol y de HDL, pero disminuye los triglicéridos, en cualquier caso, donde los niveles de adrenocorticotropina (hormona del estrés), se aumente por más de seis días.

También, se encontró que las altas concentraciones de triglicéridos están relacionadas significativamente con los niveles de colesterol total en las dos líneas (Cuadro 1). Esto se podría explicar por los altos niveles de VLDL presentes en las gallinas ponedoras, aunque la concentración de colesterol libre y esterificado dentro de las VLDL es baja (Hermier et al. 1989); entre más VLDL circule por el torrente sanguíneo más colesterol total y triglicéridos van a estar disponibles para la yema de huevo.

En conclusión, en la semana 26 de vida, las gallinas Hy-Line W-36 presentan niveles más elevados de colesterol y de triglicéridos en suero, contrario a la Línea Lohmann BrownClassic, la cual, tuvo mayor concentración de colesterol HDL. Es pertinente tener en cuenta el efecto del estrés en las aves, para evitar posibles alteraciones en los resultados, no solamente en la bioquímica sanguínea sino en la producción de huevos.

Es necesario realizar trabajos adicionales, para saber cuánto puede afectar los altos niveles de triglicéridos, de colesterol total y de colesterol HDL en la grasa del huevo, cuando la producción está disminuida. Además, se sugiere realizar es- 
tudios más profundos en cuanto a la relación del colesterol de las diferentes lipoproteínas, en el colesterol total del suero $\mathrm{y}$, al mismo tiempo, con el colesterol total de la yema de huevo y como cambia la relación de estos parámetros en las diferentes líneas, especialmente, en las diferentes etapas de la producción.

Agradecimientos: A Jorge Enrique Pérez, por la colaboración en el análisis de las muestras; a Luis Fernando Reyes González y Alex Narváez Solís, por su colaboración en el trabajo de campo. Conflicto de intereses: El manuscrito fue preparado y revisado con la participación de todos los autores, quienes declaramos que no existe ningún conflicto de interés que ponga en riesgo la validez de los resultados presentados. Fue financiado por los grupos de investigación en Ciencias Veterinarias CIENVET y BIOSALUD, de la Universidad de Caldas.

\section{BIBLIOGRAFÍA}

1. AN, B.K.; NISHIYAMA, H.; TANAKA, K.; OHTANI, S.; IWATA, T.; TSUTSUMI, K.; KASAI, M. 1997. Dietary safflower phospholipid reduces liver lipids in laying hens. Poult. Sci. (United States) 76(5):689-695.

2. ANDERSON, K.E. 2011. Comparison of fatty acid, cholesterol, and vitamin A and E composition in eggs from hens housed in conventional cage and range production facilities. Poult. Sci. 90(7):1600-1608.

3. CHOWDHURY, S.R.; CHOWDHURY, S.D.; SMITH, T.K. 2002. Effects of dietary garlic on cholesterol metabolism in laying hens. Poult. Sci. 81(12):1856-1862.

4. CHOWDHURY, S.R.; SARKER, D.K.; CHOWDHURY, S.D.; SMITH, T.K.; ROY, P.K.; WAHID, M.A. 2005. Effects of dietary tamarind on cholesterol metabolism in laying hens. Poult. Sci. 84(1):56-60.

5. ELKIN, R.G.; FREED, M.; WATKTNS, B.A.; SREBNIK, M.; KIEFT, K.A.; NEWTON, R.S. 1993. Evaluation of two novel biochemicals on plasma and egg yolk lipid composition and laying hen performance. Poult. Scie. 72(3):513-520.

6. HERMIER, D. 1997. Lipoprotein metabolism and fattening in poultry. J. Nutr. (United States). 127(5):805S808 S.

7. HERMIER, D.; CHAPMAN, J.; LECLERCQ, B. 1984. Plasma lipoprotein in fasted and refed chickens of two strains selected for high or low adiposity. J. Nutr. 114(6):1112-1121.
8. HERMIER, D.; FORGEZ, P.; WILLIAMS, J.; CHAPMAN, M.J. 1989. Alterations in plasma lipoproteins and apolipoproteins associated with estrogen-induced hyperlipidemia in the laying hen. Eur. J. Biochem. 184(1):109-118.

9. HY-LINE INTERNATIONAL. 2009. Hy-Line variety W-36 Comercial managment guide. In: Hy-Line International, ed. International, H.L., Iowa (USA). p.4.

10. KÖNIG, B.; KLUGE, H.; HAASE, K.; BRANDSCH, C.; STANGL, G.; EDER, K. 2007. Effects of clofibrate treatment in laying hens. Poult. Sci. 86(6):11871195.

11. KUDZMA, D.J.; CLAIRE, F.; DELALLO, L.; FRIEDBERG, S.J. 1975. Mechanism of avian estrogen-induced hypertriglyceridemia: evidence for overproduction of triglyceride. J. Lipid. Res. (United States). 16(2):123133.

12. LAGARRIGUE, S.; DAVAL, S.; BORDAS, A.; DOUAIRE, M. 2000. Hepatic lipogenesis gene expression in two experimental egg-laying lines divergently selected on residual food consumption. Genet Sel Evol. 32:205216.

13. LIU, X.; ZHAO, H.L.; THIESSEN, S.; HOUSE, J.D.; JONES, P.J.H. 2010. Effect of plant sterol-enriched diets on plasma and egg yolk cholesterol concentrations and cholesterol metabolism in laying hens. Poult. Sci. 89(2):270-275.

14. MONTGOMERY, R.; CONWAY, T. 1998. Bioquímica casos y texto. 6ta Ed. Harcourt Brace (Madrid). p.296383.

15. MUMMA, J.O.; THAXTON, J.P.; VIZZIER-THAXTON, Y.; DODSON, W.L. 2006. Physiological stress in laying hens. Poult. Sci. 85(4):761-769.

16. MURRAY, R.K.; MAYES, P.A.; GRANNER, D.K.; RODWELL, V.W. 2004. Harper Bioquímica ilustrada. 16ava Ed. Manual Moderno (México D.F.). p.125247.

17. NEWSHOLME, E.A.; LEECH, A.R. 1987. Bioquímica Médica. Interamericana McGraw-Hill (Madrid). p.593-632

18. NIMPF, J.; SCHNEIDER, W.J. 1991. Receptor mediated lipoprotein transport in laying hens. J. Nutri. 121(9):1471-1474. 
19. NOYAN, A.; LOSSOW, W.J.; BROT, N.; CHAIKOFF, I.L. 1964. Pathway and form of absorption of palmitic acid in the chicken. J. Lipid. Res. 5(4):538-541.

20. NOVAK, C.; SCHEIDELER, S.E. 2001. Long-term effects of feeding Flaxseed-based diets. 1. egg production parameters, components, and eggshell quality in two strains of laying hens. Poult. Sci. 80(10):14801489 .

21. PEEBLES, E.D.; BURNHAM, M.R.; WALZEM, R.L.; BRANTON, S.L.; GERARD, P.D. 2004. Effects of fasting on serum lipids and lipoprotein profiles in the egg-laying hen (Gallus domesticus). Comp. Biochem. Physiol. Mol. Integr. Physiol. 138(3):305-311.

22. SALMA, U.; MIAH, A.G.; TAREQ, K.M.A.; MAKI, T.; TSUJII, H. 2007. Effect of dietary Rhodobacter capsulatus on egg-Yolk cholesterol and laying hen performance. Poult. Sci. 86(4):714-719.

23. VIEIRA, P.M.; VIEIRA, A.V.; SANDERS, E.J.; STEYRER,
T.E.; NIMPF, J.; J., S.W. 1995. Chicken yolk contains bona fide high density lipoprotein particles. J. Lipid. Res. 36:601-610.

24. WALZEM, R.L.; HANSEN, R.J.; WILLIAMS, D.L.; HAMILTON, R.L. 1999. Estrogen induction of VLDLy Assembly in Egg-Laying hens. J. Nutr. 129(2):467S $472 S$.

25. WEGGEMANS, R.M.; ZOCK, P.L.; KATAN, M.B. 2001. Dietary cholesterol from eggs increases the ratio of total cholesterol to high-density lipoprotein cholesterol in humans: a meta-analysis. Am. J. Clin. Nutr. (USA). 73(5):885-891.

26. YIN, J.D.; SHANG, X.G.; LI, D.F.; WANG, F.L.; GUAN, Y.F.; WANG, Z.Y. 2008. Effects of dietary conjugated linoleic acid on the fatty acid profile and cholesterol content of egg yolks from different breeds of layers. Poult. Sci.. 87(2):284-290.

Recibido: Diciembre 19 de 2011

Aceptado: Febrero 8 de 2013 